\title{
Standard criteria versus Rosemont classification for EUS-diagnosis of chronic pancreatitis
}

\author{
Cristina Jimeno-Ayllón, José Ignacio Pérez-García, Carmen Julia Gómez-Ruiz, \\ Jesús García-Cano-Lizcano, Julia Morillas-Ariño, Raquel Martínez-Fernández, Lorena Serrano-Sánchez \\ and Ángel Pérez-Sola
}

Division of Digestive Diseases. Unit of Endoscopy. Hospital General Virgen de la Luz. Cuenca, Spain

\begin{abstract}
Aim: to study the possible differences in the final diagnosis of chronic pancreatitis by using standard classification described by Wiersema et al. and the new classification proposed recently by Rosemont.

Material and methods: forty-seven patients with the diagnosis of chronic pancreatitis were included in this study. The parenchymal and ductal criteria were studied, the patients were divided in two groups for Wiersema criteria: $<4$ criteria, non-diagnostic for chronic pancreatitis and $\geq 4$ criteria, diagnosis of chronic pancreatitis. The same patients were divided in four groups according to Rosemont classification: normal pancreas, indeterminate, suggestive and consistent with chronic pancreatitis. We analyzed these data with Chi-square test reported with 95\% confidence intervals (CI).

Results: in patients with chronic pancreatitis the most frequent criteria observed were lobularity in $66 \%$ of cases and pancreatic duct dilatation and calcifications in $57.4 \%$ of cases each. We found a significant statistical association between the results of both classifications $(p<0.05)$. The highest association is found in patients with more than 4 standard criteria and definitive diagnostic of chronic pancreatitis according to Rosemont classification. In patients who have less than 4 standard criteria the diagnosis is suggestive of chronic pancreatitis by using the Rosemont classification in $27.66 \%$ ( $p<0.05)$.

Conclusion: these results show that no significant statistical differences are found for patients with $>4$ criteria diagnosis by standard criteria. But $27.66 \%$ patients with less than 4 standard criteria would be suggestive according to Rosemont classification $(\mathrm{p}<$ 0.05 ). Hence, the new classification would be useful in patients with high suspicion of chronic pancreatitis with $<4$ standard criteria but with more significance such as parenchymal lithiasis, lobularity or ductal calcifications.
\end{abstract}

Key words: Chronic pancreatitis. Endoscopic ultrasound. Rosemont criteria.

Received: 20-03-11.

Accepted: 05-07-11.

Correspondence: Cristina Jimeno-Ayllón. Division of Digestive Diseases. Unit of Endoscopy. Hospital General Virgen de la Luz. C/ Hermandad de Donantes de Sangre, 1. 16002 Cuenca, Spain

e-mail: cjimenoayllon@yahoo.es
Jimeno-Ayllón C, Pérez-García JI, Gómez-Ruiz CJ, García-CanoLizcano J, Morillas-Ariño J, Martínez-Fernández R, Serrano-Sánchez L, Pérez-Sola A. Standard criteria versus Rosemont classification for EUS-diagnosis of chronic pancreatitis. Rev Esp Enferm Dig 2011; 103: 626-631.

\section{INTRODUCTION}

Chronic pancreatitis is an irreversible disease of exocrine pancreas characterized by progressive destruction of pancreatic parenchyma and remodeling processes leading to the replacement of the exocrine parenchyma by extensive fibrosis (1). These changes result in malabsorption, diabetes mellitus and severe and chronic abdominal pain (2). Moreover, it is possible the development of several complications which will determine the clinical course developing in some cases pancreatic pseudocyst (25-30\%), bile duct strictures (40-50\%), even pancreatic cancer (1-3\%). The mainstay is detecting early pancreatic changes and treating its related complications.

The diagnosis of chronic pancreatitis has been made traditionally by CT-scan or ERCP. CT-scan shows pancreatic calcification and a dilated pancreatic duct, but could misdiagnose some early chronic pancreatitis. On the other hand, ERCP is an invasive procedure that reveals advanced Cambridge criteria (3), the pancreatic changes could be detected later with ERCP than EUS $(4,5)$.

MRCP is a non-invasive procedure and a good alternative to ERCP, consequently has been used in patients with high risk of developing post-ERCP pancreatitis or with an anatomic variation secondary to pancreatic or gastric surgery $(5,6)$. The diagnosis also can be reached by 
exocrine pancreatic function tests. There are two types of testing: direct function test, which gold standard is secretin-pancreozymin test (SPT) but is not well standardized. Indirect function test are studied by pancreatouryl test, chymotrypsin or elastase- 1 estimation (7). The main drawback of this test is the low sensitivity in early and mild chronic pancreatitis and the unavailability in most of the non referral hospitals (6).

Since 1986, there are studies reporting the use of ultrasound endoscopy for the diagnosis of chronic pancreatitis. Two studies have reported that about $65 \%$ of patients with early CP detected on EUS but not on other procedures such as CT-scan, secretin function test or ERCP will eventually develop CP, which can be detected on repeated tests that were previously negative $(3,4)$.

The most frequent used classification for this purpose has been the classification described by Wiersema et al. in 1993. Wiersema described 9 pancreatic criteria: hiperechoic foci, hyperechoic strands, lobularity, cyst, calcification, main pancreatic duct (MPD) dilatation, side branch dilatation, pancreatic duct irregularity and hyperechoic duct margins $(8,9)$. All these criteria give equal value for the final score (6). In April 2007, a new classification was proposed in an international consensus meeting in Rosemont, Illinois. This new criteria gives a different value according to the criteria, establishing major and minor criteria depending on the features found (10).

A new technique, the second generation elastography has been recently developed; nevertheless there are few studies about its feasibility for CP. EUS-elastography determines the tissue hardness being useful in the differential diagnosis of pancreatic solid masses (malignant or benign inflammatory mass in $\mathrm{CP}$ ). The subjective interpretation is the primary weak point of this procedure and more studies are necessary at this point (11).

\section{MATERIALS AND METHODS}

\section{Study design}

This observational retrospective cohort study analyzed the video of patients who had undergone a EUS for suspected or established CP, between September 2007 and December 2010. The diagnosis of CP for suspected cases had been confirmed by CT-scan and/or MRI. The etiologic, demographic and clinic data were extracted from the Cuenca's Gastroenterology Database designed in 2002.

\section{Video scoring}

A set of 47 videos were observed by two EUS expert, blinded to the original diagnosis and number of chronic pancreatitis features. First, two experts interpreted the video by using the standard criteria, later both experts interpreted the same videos by using the Rosemont criteria.
Table I. EUS diagnosis of CP on the basis of Rosemont criteria

\begin{tabular}{ll}
\hline I. Consistent with chronic & $\begin{array}{l}\text { A. } 1 \text { major A feature }+\geq 3 \text { minor } \\
\text { pancreatitis }\end{array}$ \\
& features \\
& B. 1 major A feature + major B feature \\
& C. 2 major A features \\
II. Suggestive of CP & A. 1 major A feature $+<3$ minor \\
& features \\
& B. 1 major B feature $+\geq 3$ minor \\
& features \\
& C. $\geq 5$ minor features (any) \\
& A. 3 to 4 minor features, no major \\
III. Indeterminate for CP & features \\
& B. Major B feature alone or with $<3$ \\
minor features \\
$\leq 2$ minor features, no major features
\end{tabular}

EUS was done under conscious sedation using midazolam and dolantine. A Hitachi EUB-6500 ultrasound console was used for the recording with the endoscopes Pentax electronic radial EG-3670 URK echoendoscope (Pentax, Tokyo, Japan) in 16 cases $(34.04 \%)$ and Pentax curved-linear array EG-3870 UTK echoendoscope (Pentax Europe $\mathrm{GmbH}$, Hamburg, Germany) in 31 cases $(65.96 \%)$.

It was analyzed the type and the number of criteria according to standard classification described by Wiersema et al. (hyperecoic foci, hyperecoic strands, cysts, lobularity, calcifications, main duct dilatation, main duct irregularity, visible side branches and hyperechoic main duct walls) defining as diagnostic of chronic pancreatitis the presence of $\geq 4$ criteria and non diagnostic $<4$ criteria (9). Therefore the Rosemont criteria were used (major criteria: hyperecoic foci with shadowing and main pancreatic duct calculi, lobularity with honeycombing. Minor criteria included cysts, dilated ducts $\geq 3.5 \mathrm{~mm}$, irregular pancreatic duct contour, dilated side branches $\geq 1 \mathrm{~mm}$, hyperechoic duct wall, strands, nonshadowing hyperechoic foci, and lobularity with noncontiguous lobules), defining 4 groups of patients after studying the major and minor criteria: normal pancreas, indeterminate, suggestive and consistent with chronic pancreatitis (10) (Table I).

\section{Statistical methods}

Percentages are reported for etiology, clinical characteristics of patients and features of CP. Chi-square test was used to analyze the concordance between standard criteria and Rosemont classification with $95 \%$ confidence intervals. A p-value $<0.05$ was considered statistically significant. SPSS version 11.0 software was used for all analyses. 
Table II. Clinical characteristics

\begin{tabular}{ll}
\hline Clinical feature & $n=47$ \\
\hline Age (years \pm SD) & $54 \pm 11.57$ \\
Gender & \\
Male & $38(80.9 \%)$ \\
Female & $9(19.1 \%)$ \\
Etiology & \\
Alcohol & $29(67.4 \%)$ \\
Unknown & $13(30.2 \%)$ \\
Hypertriglyceridemia & $1(2.3 \%)$ \\
Risk factors & \\
Alcohol and tobacco & $19(44.2 \%)$ \\
Alcohol and hypertriglyceridemia & $3(7 \%)$ \\
Alcohol, tobacco and hypertriglyceridemia & $4(9.3 \%)$ \\
Alcohol, tobacco and renal chronic insufficiency & $1(2.3 \%)$ \\
Only alcohol & $8(18.6 \%)$ \\
Only tobacco & $1(2.3 \%)$ \\
None & $6(14 \%)$ \\
Previous acute pancreatitis & $26(60.5 \%)$ \\
Acute recurrent pancreatitis & $19(44.2 \%)$ \\
Symptoms & \\
Abdominal pain & $16(36.4 \%)$ \\
Diarrhea & $3(7 \%)$ \\
Diabetes mellitus & $19(45.2 \%)$ \\
CT-scan & \\
Concordant & $22(73.3 \%)$ \\
Discordant & $8(26.7 \%)$ \\
MRI & \\
Concordant & $20(90.9 \%)$ \\
Discordant & $2(9.1 \%)$ \\
\hline
\end{tabular}

\section{RESULTS}

A total of 47 patients underwent EUS between September 2007 and December 2010 for:

- Established CP $(n=14 ; 34.1 \%)$ and confirmed by CTscan and/or MRI.

- Acute recurrent pancreatitis $(\mathrm{n}=8 ; 19.5 \%)$.

- Incidental pancreatic cyst $(\mathrm{n}=5 ; 12.2 \%)$.

- Acute pancreatitis $(\mathrm{n}=4 ; 9.8 \%)$.

- Abdominal pain $(n=4 ; 9.8 \%)$.

- Others: jaundice $(\mathrm{n}=2 ; 4.9 \%)$, duct Wirsung irregularity of unknown origin $(n=1 ; 2.4 \%)$, calcifications detected by other image test $(\mathrm{n}=1 ; 2.4 \%)$ and diarrhea $(\mathrm{n}=1 ; 2.4 \%)$.

There were 38 males and 9 females and the mean age was $54 \pm 11.57$ years. The etiologic and clinical characteristics are showed in table II.

In the first analyses of videos by the expertise standard score were used, detecting the following features: hyperechoic foci in $23.4 \%(\mathrm{n}=11)$, hyperechoic strands in $51.1 \%$ $(\mathrm{n}=24)$, lobularity in $66 \%(\mathrm{n}=31)$, cysts in $19.1 \%(\mathrm{n}=$ 9), calcifications in $57.4 \%(\mathrm{n}=27)$, main pancreatic duct dilatation in $57.4 \%(\mathrm{n}=27)$, side branch dilatation in $19.1 \%$ $(n=9)$, main pancreatic duct irregularity in $44.7 \%(n=21)$ and hyperechoic main duct margins in $48.9 \%(n=23)$ of cases. The majority of patients had 4 criteria for diagnosing chronic pancreatitis $(\mathrm{n}=15,31.9 \%)$ followed in frequency by 5 criteria $(\mathrm{n}=9,19.1 \%)$.

The two endosonographers analyzed the same videos a second time by using the Rosemont score, there were described: 29 major A features, 25 major B features, 46 minor parenchymal features and 69 minor ductal features. With these criteria four groups were classified according to Rosemont classification: normal pancreas $6.38 \%$, indeterminate for CP $12.77 \%$, suggestive of CP $40.42 \%$ and consistent with $\mathrm{CP} 40.42 \%$.

The Chi-square test was used to compare the features found in both groups, showing a statistically significant association between the results of standard and Rosemont scoring ( $p<0.05)$. The highest association was found for patients with more than 4 standard criteria and definitive diagnostic of chronic pancreatitis according to Rosemont classification. In patients who have less than 4 standard criteria and the diagnostic was suggestive with the Rosemont classification a statistically association was found $(\mathrm{p}<0.05)$.

Also, there were analyzed other parenchymal features usually not used for EUS-diagnosis of chronic pancreatitis such as parenchymal atrophy that was detected in $45.7 \%$ and mass effect revealed in $21.3 \%$ of cases.

\section{DISCUSSION}

Despite the studies about EUS diagnosis of CP, the criteria used for its definition have not been well defined $(12,13)$. EUS demonstrates to be useful for early diagnosis of $\mathrm{CP}$ being able to identify features missing on CTscan, ERCP or MRCP $(5,6,14-16)$.

In our study, we compared the number and type of features in all patients undergoing EUS with new or previously diagnosis of CP. Our data show that most patients have an established CP $(\mathrm{n}=14 ; 34.1 \%)$ followed by acute recurrent pancreatitis $(n=8 ; 19.5 \%)$ at the time of performance EUS.

The main age of patients of our study was $54 \pm 11.57$ years, this age is not advanced enough to be changes generate by the elderly (17). The predominant sex was male $(80.9 \%)$ and the most frequent etiology was alcohol consumption $(67.4 \%)$.

The unavailability of functional test for $\mathrm{CP}$ diagnosis in our hospital forced us to corroborate the diagnosis of $\mathrm{CP}$ with other imaging test such as CT-scan or MRI (18), confirming a higher concordance with MRCP than CT-scan (90.9 vs. $73.3 \%$ ).

The aim of our study was to analyze the possible differences in the final diagnostic of chronic pancreatitis by using the standard classification described by Wiersema et al. and the new classification proposed recently in Rosemont (10).

The feature most frequent found in the patients of our study was the lobularity (66\%), followed by MPD dilation $(57.4 \%)$ and calcifications (57.4\%). Most of our patients 
$(63.82 \%)$ presented lobularity with honeycombing (a major $\mathrm{B}$ feature). Another frequent feature is the hyperechoic foci with shadowing (major A). Therefore, lobularity and parenchymal calcifications are higher importance (major criteria) using the Rosemont than standard criteria.

When we compared both classifications using the Chisquare test, a statistical difference is showed for patients with less than 4 standard criteria and features suggestive of $\mathrm{CP}$ with Rosemont classification $(\mathrm{p}<0.05)$. However no significant statistical differences are found for patients with $\geq 4$ criteria diagnostic by standard criteria. As shown in figure 1,13 patients (27.66\%) with less than 4 standard criteria will be suggestive of $\mathrm{CP}$ with the Rosemont classification $(\mathrm{p}<0.05)$.

In contrast, the last multicenter study concerning EUS for CP diagnosis, Stevens et al., demonstrates lower detection rates with Rosemont scoring compared with standard scoring (12).

These results do not reveal the better results obtained with the new Rosemont classification but suggest a high suspicion of CP. For these patients we could perform other diagnostic test for clarifying the pancreatic alterations. A pancreatic function test with SPT, MRCP in patients without contraindications for this procedure, ERCP in patients without anatomical abnormalities and CT-scan might be useful in theses situations (6) as we propose in the algorithm of the figure 2. In addition, these morphologic and functional findings may be enhanced in conjunction with clinical symptoms (1).

Up to now, few studies combining endoscopic ultrasound and secretin endoscopic pancreatic function test (ePFT) have been reported. This procedure detects exocrine insufficiency in association with early and late stages of pancreatic fibrosis. The results at this point (Stevens et al.) are

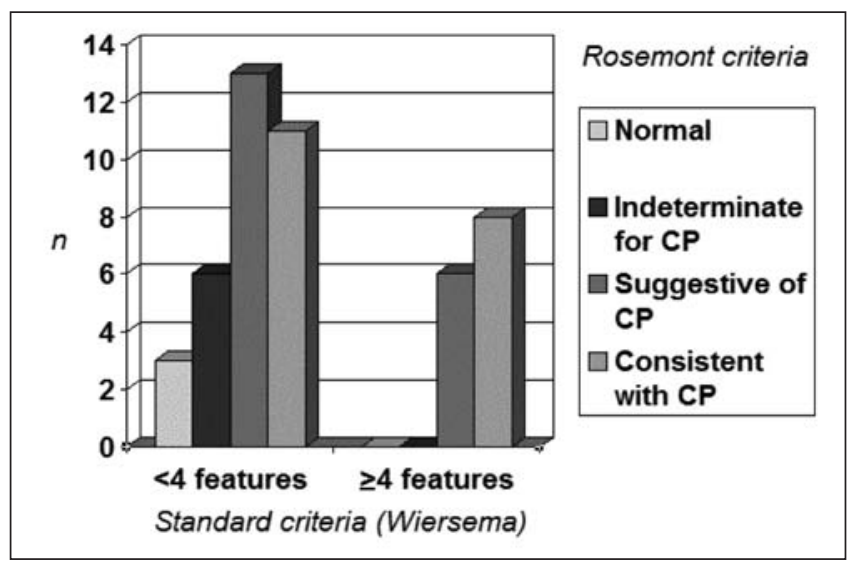

Fig. 1. Standard criteria compared with Rosemont criteria in a cohort of 47 patients.

promising and we could use in a future EUS in combination with secretin function test (14).

One limitation of this study is the reduced number of patients $(n=47)$ and they are predominantly alcoholic etiology. A second limitation is the known poor interobserver agreement described in the EUS studies for CP $(1,12)$ but not analyzed in this study.

We conclude with our study that no significant difference is found for patients with $\geq 4$ criteria diagnosing by standard criteria. But 13 patients with less than 4 standard criteria would be suggestive according to Rosemont classification $(27.66 \%$; $\mathrm{p}<0.05)$. Hence, the new classification would be useful in patients with high suspicion of chronic pancreatitis (suspect clinic manifestations, pan-

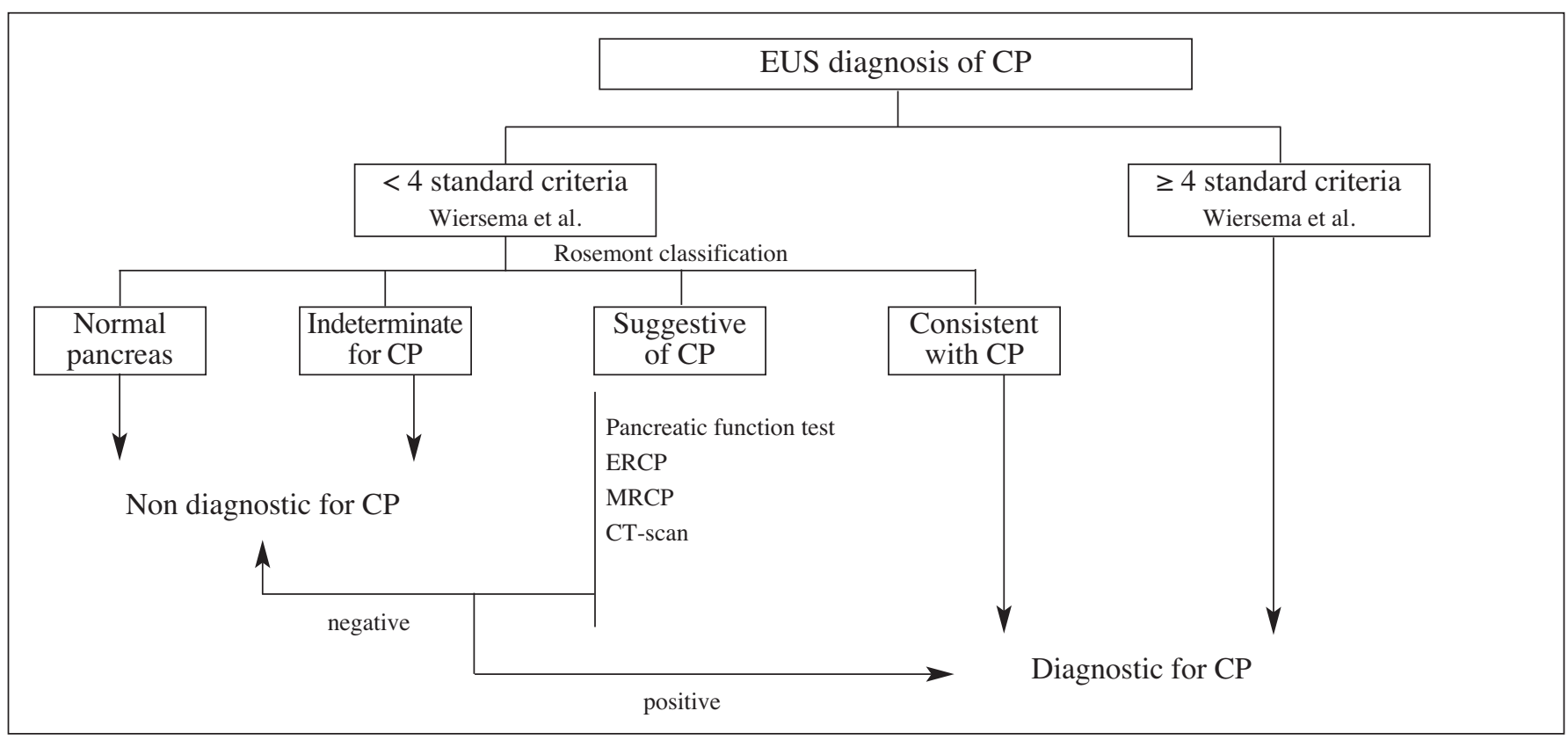

Fig. 2. Algorithm proposed for the EUS diagnosis of chronic pancreatitis. 


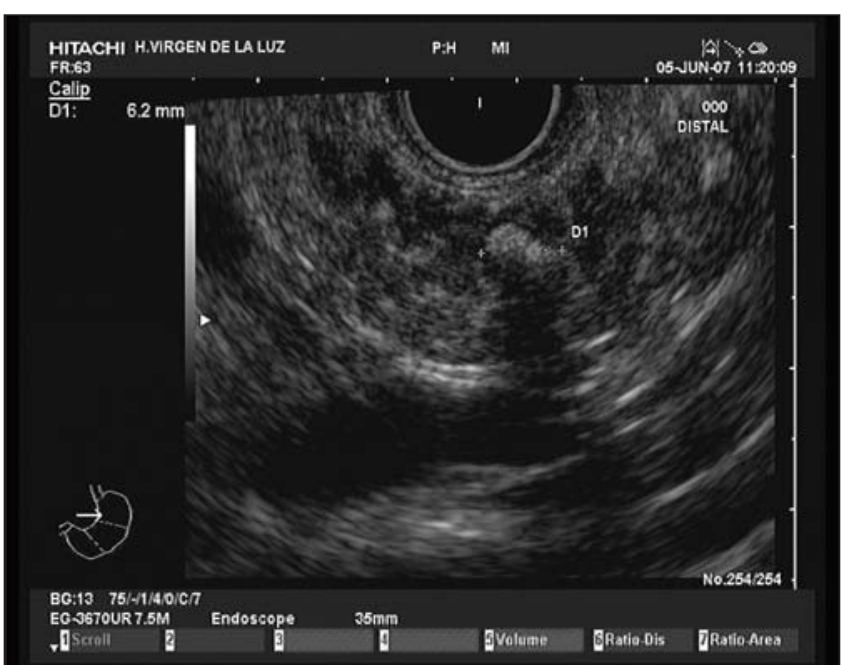

Fig. 3. EUS image of the pancreatic body containing a 6,2 $\mathrm{mm}$ intraductal stone with dilation and irregularity of the main pancreatic duct.

creatic test, ERCP, CT-scan or MRCP findings) with $<4$ standard criteria but with more significance such as parenchymal lithiasis, lobularity or ductal calcifications (Figs. 3 and 4).

\section{REFERENCES}

1. Gardner TB, Levy MJ. EUS diagnosis of chronic pancreatitis. Gastrointest Endosc 2010;71(7):1280-9.

2. Etemad B, Whitcomb DC. Chronic pancreatitis: diagnosis, classification, and new genetic developments. Gastroenterology 2001;120(3):682707.

3. Gleeson FC, Topazian M. Endoscopic retrograde cholangiopancreatography and endoscopic ultrasound for diagnosis of chronic pancreatitis. Curr Gastroenterol Rep 2007;9(2):123-9.

4. Kahl S, Glasbrenner B, Leodolter A, Pross M, Schulz HU, Malfertheiner P. EUS in the diagnosis of early chronic pancreatitis: a prospective follow-up study. Gatrointest Endosc 2002;55(4):507-11.

5. Morris Stiff G, Webster P, Frost B, Lewis WG, Puntis MC, Roberts SA. Endoscopic Ultrasound identifies chronic pancreatitis when other imaging modalities have been non-diagnostic. JOP 2009;10(3):280-3.

6. Witt H, Apte MV, Keim V, Wilson JS. Chronic pancreatitis: challenges and advances in pathogenesis, genetics, diagnosis, and therapy. Gastroenterology 2007;132(4):1557-73.

7. Lankisch PG. The problem of diagnosing chronic pancreatitis. Dig Liver Dis 2003;35(3):131-4

8. Wallace MB. Chronic pancreatitis. Gastrointest Endosc 2009;69(Supl. 2):S117-120

9. Wiersema MJ, Hawes RH, Lehman GA, Kochman ML, Sherman S, Kopecky KK. Prospective evaluation of endoscopic ultrasonography and endoscopic retrograde cholangiopancreatography in patients with

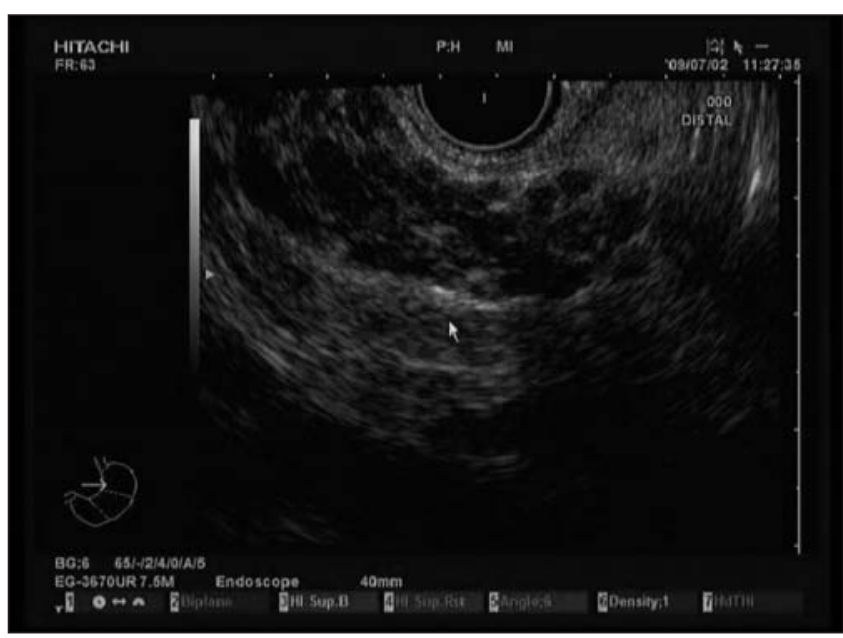

Fig. 4. Pancreatic parenchyma demonstrating honeycombing lobularity

chronic abdominal pain of suspected pancreatic origin. Endoscopy 1993;25(9):555-64.

10. Catalano MF, Sahai A, Levy M, et al. EUS-based criteria for the diagnosis of chronic pancreatitis: the Rosemont classification. Gastrointest Endosc 2009;69(7):1251-61

11. Iglesias García JJ, Lariño Noia J, Alvarez Castro A, Cigarran B, Dominguez Muñoz JE. Second-generation endoscopic ultrasound elastography in the differential diagnosis of solid pancreatic masses. Pancreatic cancer vs. inflammatory mass in chronic pancreatitis. Rev Esp Enferm Dig 2009;101(10):723-30.

12. Stevens T, Lopez R, Adler G, Al-Haddad MA, Conway J, Dewitt JM, et al. Multicenter comparison of the interobserver agreement of standard EUS scoring and Rosemont classification scoring for diagnosis of chronic pancreatitis. Gastrointest Endosc 2010;71(3):519-26.

13. Catalano MF, Hernandez LV, Kaul V, Guda NM, Pezanoski JP, Ramasamy D, et al. EUS diagnosis of chronic pancreatitis (CP): comparison of the current criteria $v s$. the new "Rosemont Criteria" developed by an international consensus conference. Gastrointest Endosc 2008;67 (5):AB215.

14. Stevens T, Dumot JA, Parsi MA, Zucarro G, Vargo JJ. Combined Endoscopic ultrasound and secretin endoscopic pancreatic function test in patients evaluated for chronic pancreatitis. Dig Dis Sci 2010; 55(9):2681-7.

15. Catalano MF, Kaul V, Pezanoski J, Guda N, Geenen JE. Long term outcome of endosonographically detected minimum criteria for chronic pancreatitis (MCCP) when conventional imaging and functional testing are normal. Gastrointest Endosc 2007;65(5):AB120.

16. Kaul V, Catalano M. EUS and chronic pancreatitis: seeing is believing? Gastrointest Endosc 2007;66(3):510-2.

17. Larghi A, Petrone MC, Galasso D, Arcidiacono PG. Endoscopic ultrasound in the evaluation of pancreatobiliary disorders. Dig Liver Dis 2010;42(1):6-15.

18. Poves E, Del Pozo D, Tabernero S, Bardina A, Martínez P, Castillo MC. Impacto clínico de la introducción de la ultrasonografía endoscópica (USE) alta en un hospital de área. Rev Esp Enferm Dig 2010; 102(12):698-703 